\title{
Biomarker Candidates of Chlamydophila pneumoniae Proteins and Protein Fragments Identified by Affinity-Proteomics Using FTICR-MS and LC-MS/MS
}

\author{
Iuliana Susnea, ${ }^{1}$ Sebastian Bunk, ${ }^{2}$ Albrecht Wendel, ${ }^{2,3}$ Corinna Hermann, ${ }^{2}$ \\ Michael Przybylski ${ }^{1}$ \\ ${ }^{1}$ Laboratory of Analytical Chemistry and Biopolymer Structure Analysis, Department of Chemistry, University of Konstanz, \\ 78457 Konstanz, Germany \\ ${ }^{2}$ Laboratory of Biochemical Pharmacology, Department of Biology, University of Konstanz, Konstanz, Germany \\ ${ }^{3}$ Present address: Pharmacogenomics Center, University of Tübingen, Ottfried-Müller-Strasse 27, 72070 Tübingen, Germany
}

\begin{abstract}
We report here an affinity-proteomics approach that combines 2D-gel electrophoresis and immunoblotting with high performance mass spectrometry to the identification of both full length protein antigens and antigenic fragments of Chlamydophila pneumoniae (C. pneumoniae). The present affinity-mass spectrometry approach effectively utilized high resolution FTICR mass spectrometry and LC-tandem-MS for protein identification, and enabled the identification of several new highly antigenic $C$. pneumoniae proteins that were not hitherto reported or previously detected only in other Chlamydia species, such as Chlamydia trachomatis. Moreover, high resolution affinity-MS provided the identification of several neo-antigenic protein fragments containing $\mathrm{N}$ - and C-terminal, and central domains such as fragments of the membrane protein Pmp21 and the secreted chlamydial proteasome-like factor (Cpaf), representing specific biomarker candidates.
\end{abstract}

Keywords: Affinity-proteomics, Chlamydia pneumoniae, Protein biomarker candidates, High resolution mass spectrometry, LC- tandem mass spectrometry, Neoantigenic protein fragments

\section{Introduction}

(1. pneumoniae is an important human respiratory pathogen that causes approximately $5 \%$ of all cases of bronchitis and is believed to be responsible for about $10 \%$ of community-acquired pneumonia cases [1]. C. pneumoniae

Iuliana Susnea and Sebastian Bunk contributed equally to this work.

Electronic supplementary material The online version of this article (doi:10.1007/s13361-011-0082-3) contains supplementary material, which is available to authorized users.

Correspondence to: Michael Przybylski; e-mail: Michael.Przybylski@ uni-konstanz.de has a two-phase development cycle alternating between a metabolically inactive, but infectious form, elementary bodies (EB), and a noninfectious active form, reticulate bodies (RB) [1]. The bacterium uses human host cells for replication to which EB adhere and are phagocytosed 72 to $96 \mathrm{~h}$ post-infection in which time EB develop into RB [2]. C. pneumoniae persisting in the host following primary infection has been associated with atherosclerosis leading to cardiovascular diseases [3]. Albeit investigations for more than $15 \mathrm{y}$, the role of $C$. pneumoniae infection still remains controversial, with the lack of reproducible serodiagnosis being a major drawback [4].

In previous studies, only limited molecular knowledge has been obtained about the immunodominant antigens in 
Table 1. Antigenic proteins identified by MALDI-FTICR- MS (A) and LCtandem mass spectrometry (B). Numbers of gel spots are as in Supplementary Figure 1

\begin{tabular}{lcccc}
\hline Protein name/acc. no. $^{\mathrm{a}}$ & Spot no. & Mass (kDa) & pI & MS method \\
\hline Omp2/P23700 & $3^{\mathrm{b}}$ & 61.63 & 6.0 & $\mathrm{~A}$ \\
DnaK/P27542 & $7^{\mathrm{c}}$ & 71.42 & 5.0 & $\mathrm{~A}$ \\
CpB1072/Q9Z6M7 & 16 & 21.87 & 5.6 & $\mathrm{~A}, \mathrm{~B}$ \\
Pmp6/Q9Z899 & 35 & $145.97^{\mathrm{d}}$ & $5.3^{\mathrm{d}}$ & $\mathrm{A}$ \\
GAPDH/Q9Z7T0 & 47 & 37.22 & 6.3 & $\mathrm{~A}, \mathrm{~B}$ \\
ClpP1/Q9Z832 & 80 & 21.06 & 5.6 & $\mathrm{~A}, \mathrm{~B}$ \\
CpB0756/Q9Z7H7 & 89 & 68.18 & 4.9 & $\mathrm{~A}, \mathrm{~B}$ \\
\hline
\end{tabular}

a Accession numbers are from SWISS-PROT or TrEMBL database.

${ }^{\mathrm{b}}$ Omp2 was identified in protein spots 3 and 1.

${ }^{\mathrm{c}}$ DnaK was identified in protein spots 7 and 6 .

d Spot 35 represents a fragment of Pmp6 protein, which is separated at smaller molecular mass $(\sim 60 \mathrm{kDa})$ and slightly basic $\mathrm{pH}(\sim 7)$.

C. pneumoniae. The microimmunofluorescence (MIF) test, based on whole EB, has been employed as a standard for serology but presents several disadvantages such as cross-reactivity between different Chlamydia species and high interlaboratory variations [5]. Moreover, the MIF test does not correlate with the presence of $C$. pneumoniae in the host, nor differentiate between past and persistent infections [6].

A recent study on Chlamydia trachomatis by SanchezCampillo et al. employing an immunoproteomics approach, $\mathrm{N}$-terminal sequencing and homology search provided the identification of a few immunoreactive proteins [7]. In a previous study, an affinity-proteomics approach combined with FTICR-MS with high specificity and reproducibility has been developed and applied for the identification of $C$. pneumoniae antigenic structures relevant for serodiagnosis [8]. The combination of 2-DE immunoproteomics with FTICR-MS and LC-MS/MS is shown here to be highly successful in identifying several hitherto unknown antigens and, particularly, the identification of several neo-antigenic protein fragments. These results provide new and additional evidence for the specific description of biomarker candidates and design of possible therapeutic lead structures.

Table 2. Antigenic protein fragments identified by MALDI-FTICR- MS (A) and LC-tandem mass spectrometry (B). Spot numbers are according to Supplementary Figure 1

\begin{tabular}{lcccc}
\hline Protein name/acc. no. & Spot no. & Mass (kDa) & pI & MS method \\
\hline Cpaf-c/Q9Z6P3 & 26 & $69.34^{\mathrm{b}}$ & $5.5^{\mathrm{b}}$ & $\mathrm{A}, \mathrm{B}$ \\
Cpaf-n/Q9Z6P3 & 44 & $69.34^{\mathrm{b}}$ & 5.5 & $\mathrm{~A}, \mathrm{~B}$ \\
Pmp21-m/Q9Z6U5 & 9 & $169.41^{\mathrm{c}}$ & 4.8 & $\mathrm{~A}, \mathrm{~B}$ \\
Pmp21-c/Q9Z6U5 & 10 & $169.41^{\mathrm{c}}$ & 4.8 & $\mathrm{~A}, \mathrm{~B}$ \\
Pmp21-n/Q9Z6U5 & 36 & $169.41^{\mathrm{c}}$ & 4.8 & $\mathrm{~A}$
\end{tabular}

\footnotetext{
${ }^{a}$ Accession numbers are from SWISS-PROT or TrEMBL database.

${ }^{\mathrm{b}}$ Cpaf-n fragment has ca. $24 \mathrm{kDa}$ and Cpaf-c approximately $40 \mathrm{kDa}$ and a pI of 5 .

${ }^{\mathrm{c}}$ The molecular mass and the $\mathrm{pI}$ are for the full length Pmp 21 protein. The $\mathrm{N}$-terminal part has ca. $70 \mathrm{kDa}$ (spot 36 ), the middle part $\sim 55 \mathrm{kDa}$ (spot 9) and the C-terminal domain $\sim 45 \mathrm{kDa}$ (spot 10).
}

\section{Experimental}

\section{D- Gel Electrophoresis and Immunoblotting}

Sera from 39 human donors were tested for antibodies against $C$. pneumoniae, $C$. trachomatis, and $C$. psittaci using the MIF assay (Savyon Diagnostics, St. Ashdod, Israel) [8]. One serum tested positive for $C$. trachomatis and was excluded from the study. C. pneumoniae TW-183 were cultured as previously described [8].

Chlamydia samples were purified using the 2D Clean-up Kit (Amersham Biosciences, Uppsala, Sweden) and applied overnight on $17 \mathrm{~cm}$ IPG strips ( $\mathrm{pH}$ range 3-10 NL) using a passive in-gel rehydration method, as described [8]. Following separation, proteins were visualized with sensitive colloidal Coomassie staining [9], and gels were scanned with a Bio-Rad (Laboratories GmbH, München, Germany) GS-710 imaging densitometer. All separations were performed in pairs of two gels, each run involving two electrophoretic cells, with three gels used for immunoblotting and one gel for mass spectrometric identification of antigenic proteins.

Following electrophoretic separation 2D gels were electroblotted for $2 \mathrm{~h}$ at $60 \mathrm{~V}$ onto nitrocellulose membranes using a WEB-M tank blotter (PEQLAB, Erlangen, Germany). Immunoblotting was performed as previously described [8], and electronic image files matched with the 2DE maps of the Coomassie-stained gels. For the analysis of the immunoblots, the maximum intensity of each spot was determined using the AIDA software package (Raytest/Fuji, Straubenhardt, Germany) [8].

\section{Mass Spectrometry}

Spots were excised from the gels, subjected to tryptic in-gel digestion according to [10], and elution fractions desalted with C18 OMIX Pipette Tips (Varian, Walnut Creek, CA, USA.). MALDI-FTICR-MS peptide mapping was performed with a Bruker APEX II FTICR spectrometer equipped with an actively shielded 7T superconducting magnet (Bruker Daltonik, Bremen, Germany) as previously described [8]. Monoisotopic masses of singly charged ions generated by XMASS were directly used for database search [8] (http://www. matrixscience.com; and http://prowl.rockefeller.edu/).

LC-MS/MS analysis was performed on an Esquire 3000+ ion trap instrument (Bruker Daltonik) equipped with an atmospheric pressure ESI source, using nitrogen as both the nebulizing and drying gas. The instrument was operated in the data-dependent mode with MSMS acquisition upon a predetermined threshold; six microscans were collected at automatic gain control for each full MS scan and 20 microscans for each MS/MS scan, with a maximum accumulation time of $200 \mathrm{~ms}$. An Agilent-1100 HPLC was operated at $50 \mu \mathrm{L} / \mathrm{min}$, using binary elution of (A) $(0.2 \%$ aqueous formic acid) to $55 \%$ (B) $(0.2 \%$ formic acid in acetonitrile) over $90 \mathrm{~min}$, with a $10 \mathrm{~cm} \times$ $1 \mathrm{~mm}$ Discovery Bio Wide Pore C18 column (Sigma-Aldrich Chemie $\mathrm{GmbH}$, München, Germany). MS data were externally calibrated and analysed using the Bruker Data Analysis 
(a)

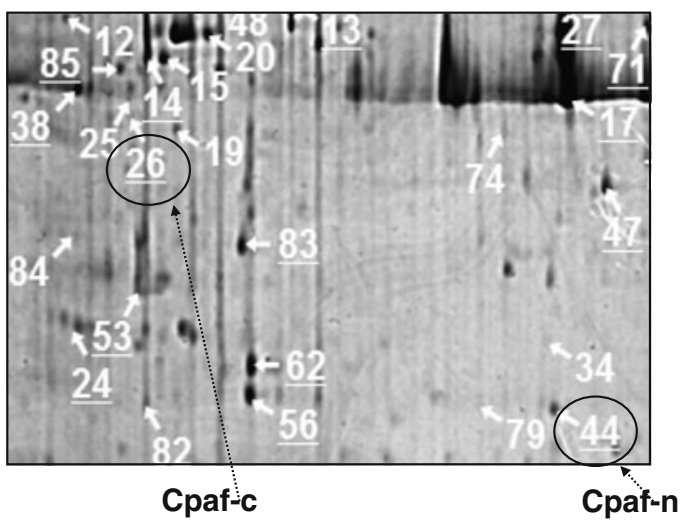

\section{Cpaf-n}

$\sim 24 \mathrm{kDa}$

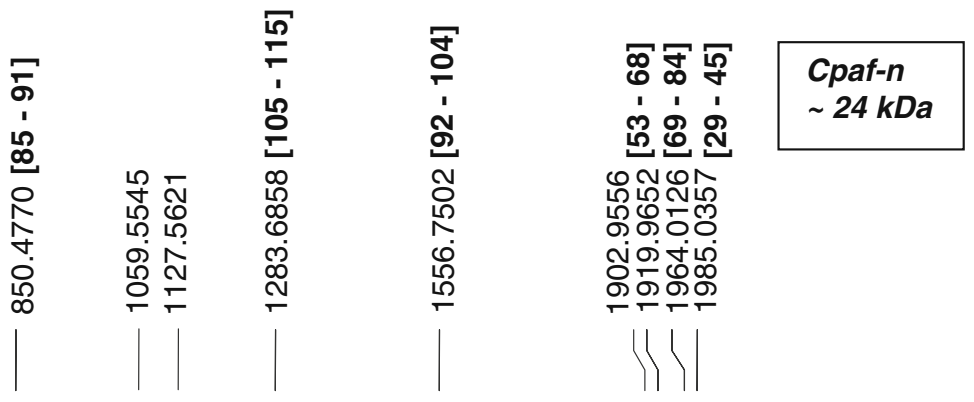

(b)

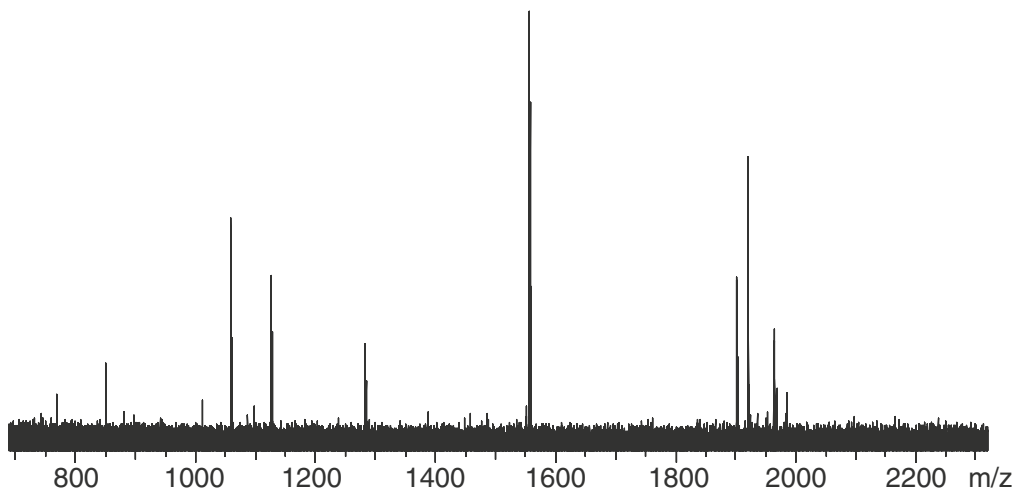

MS/MS, m/z 638.8 (2+)

(c)

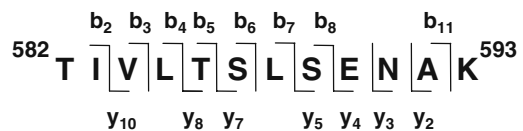

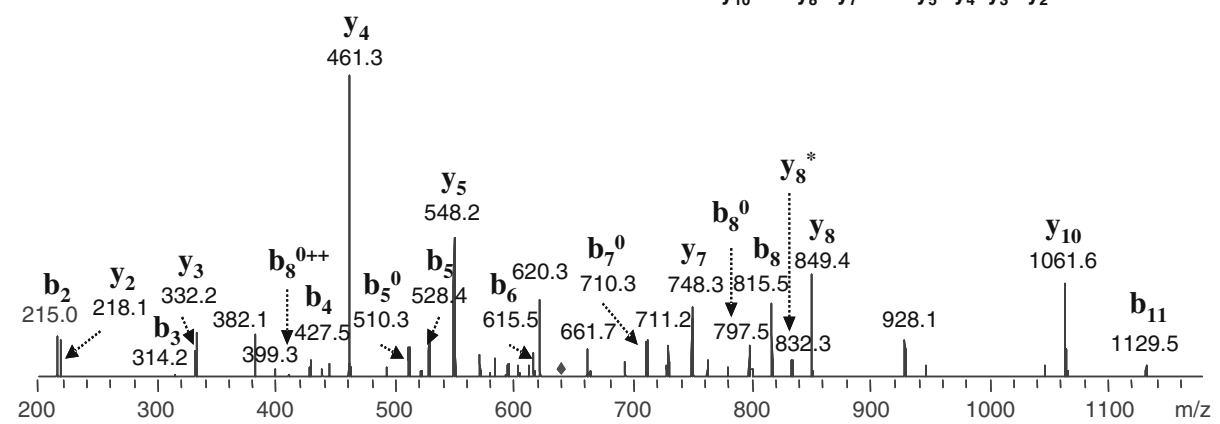

Figure 1. Identification of Cpaf (Chlamydial protease/proteasome like-activity factor) protein fragments. (a) 2D-gel section containing labeled spots 26 (Cpaf-c) and 44 (Cpaf-n). (b) MALDI-FTICR-MS of Cpaf-n tryptic peptides from spot 44; identified peptides are labeled, partial sequences are marked with brackets. (c) LC-MS/MS identification of the Cpaf-c fragment (spot 26); MS/MS of $m / z 638.8(2+)$, with $y$ and $b$ ions assigned (*: loss of $\mathrm{NH}_{3} ;{ }^{0}$ : loss of $\mathrm{H}_{2} \mathrm{O}$ ) 
software, and data saved in a *.mgf file and searched (among Other Bacteria sequence entries) against the NCBInr database.

\section{Results and Discussion}

\section{Identification of C. pneumoniae Antigens by Immunoproteomics-Mass Spectrometry}

C. pneumoniae antigens were detected in four gels, processed in each $2 \mathrm{DE}$ run under identical conditions and subjected to immunoblotting and mass spectrometry. Approximately 600 protein spots were separated within a m.w. range of ca. 15 to $130 \mathrm{kDa}$ (Supplementary Figure 1). Among the proteins visualised, 89 spots were detected as immunoreactive, and reactivities estimated based on corresponding intensities among all sera, as previously described [8].

For identification of proteins, gel spots were excised, digested with trypsin, and the proteolytic peptides analyzed by MALDI-FTICR-MS and LC- tandem mass spectrometry. Initially, proteins from 42 spots showing consistent antigenic reactivity were examined. A total of $22 C$. pneumoniae proteins (from 28 protein spots) and six human proteins were identified (Supplementary Table 1). Human proteins, also found in previously reported proteome maps [2], are likely resulting from contamination by the Hep-2 cells used in the C. pneumoniae culture. Eight $C$. pneumoniae proteins (from nine protein spots) were identified by both MALDI-FTICR-MS and LCMS/MS analysis, and are underlined in Supplementary Figure 1.

New proteins identified (not found in [8]) of substantial antigenic reactivity are summarized in Table 1 (shown underlined and in red in the 2D-gel, Supplementary Figure 1). The application of LC-tandem- MS in the present study was found to effectively extend the previous MALDIFTICR-MS data (Table 1). Protein spots 3, 7, 16, 35, 47, 80, 89 were identified as $C$. pneumoniae proteins, while spots 55, 62, 70, and 71 were human proteins. All identified $C$. pneumoniae proteins are summarized in Supplementary Table 1. For two of the eight proteins identified by LCMS/MS (Pmp21-c and Cpaf-c), single peptide masses provided individual ion scores exceeding the identity or homology threshold for unambiguous identification.

\section{Identification of Neo-antigenic Protein Fragments by High Resolution Affinity-Mass Spectrometry}

In addition to several antigenic full length proteins, new, specific neo-antigenic protein fragments were identified in this study by affinity-proteomics using LC-MS/MS (Table 2). Two polymorphic membrane proteins, Pmp6 and Pmp21, that contain an autotransporter domain and thus can pass the outer bacterial membrane, were identified both as full length proteins and as specific truncation forms. Protein spot 45 of ca. $130 \mathrm{kDa}$ was identified as full length Pmp6 ([8]), and in the present study as a $60 \mathrm{kDa}$ C-terminal fragment (spot 35) with high antigenic reactivity. For Pmp21, the largest of the 21 polymorphic C. pneumoniae membrane proteins, three fragments were identified with molecular masses of ca. 70, 55 , and $45 \mathrm{kDa}$, respectively, representing an N-terminal fragment (Pmp21-n; spot 36), a middle domain fragment (Pmp21-m; spot 9), and a C-terminal fragment (Pmp21-c; spot 10). With purified RB, no C-terminal Pmp21 fragment was found, while in purified $\mathrm{EB}$ only a $47 \mathrm{kDa} \mathrm{C}$-terminal fragment has been previously identified $[2,11]$. In contrast, the present combination approach of immunoblotting and high resolution mass spectrometry provided the unequivocal identification of all three Pmp21 fragments (Table 2).

The chlamydial protease/proteasome-like activity factor, Cpaf was identified as a further example where only neoantigenic fragments were isolated by 2-DE and identified (Table 2). Only a few secreted proteins have been hitherto identified for $C$. pneumoniae. In the present study, we identified both a $\mathrm{C}$ - and $\mathrm{N}$-terminal antigenic fragment: a basic $24 \mathrm{kDa}$ fragment (Cpaf-n; pI 7) and an acidic ca. $40 \mathrm{kDa}$ fragment (pI 5; Cpaf-c). The latter fragment had not been previously detected [12], possibly due to its comigration with the RpoA protein (Spot 14, Supplementary Figure 1). Figure 1 shows the 2D- gel spots of the Cpaf fragments, spot 26 (Cpaf-c) and spot 44 (Cpaf-n), together with the MALDI-FTICR-MS analysis and the LC-MS/MS data for the C-terminal part of Cpaf.

\section{Conclusion}

In this study, an affinity-proteomics approach in combination with high performance mass spectrometry, MALDIFTICR-MS, and LC-MS/MS demonstrated as an efficient tool to identify antigenic structures of $C$. pneumoniae relevant for serodiagnosis. In addition to previously described $C$. pneumoniae proteins, seven new reactive proteins were identified here (Table 1), which may represent specific biomarkers for $C$. pneumoniae infections. High resolution affinity- mass spectrometry proved to be a particularly efficient tool for the identification of neoantigenic protein fragments of two proteins, the full length precursor proteins of which lack antigenicity, the membrane protein Pmp21, and the chlamydial proteasome like-activity factor Cpaf (Table 2). Thus, the highly antigenic proteins identified here should be useful to (1), enable the development of more standardized serodiagnosis techniques capable to discriminate between past and persistent infections; (2), clarify the role of $C$. pneumoniae in chronic inflammatory diseases; and (3), establish biomarker candidates for future drug development.

\section{Acknowledgments}

The authors acknowledge support in part for this work by the Deutsche Forschungsgemeinschaft, Bonn, Germany (FG DNA- and Oligosaccharide Chips-Analyse sekundärer Genprodukte and FG- 753) and by the DAAD, Bonn, Germany. 


\section{References}

1. Kuo, C.C., Jackson, L.A., Campbell, L.A., Grayston, J.T.: Chlamydia pneumoniae (TWAR). Clin. Microbiol. Rev. 8, 451-461 (1995)

2. Vandahl, B.B., Birkelund, S., Christiansen, G.: Proteome analysis of Chlamydia pneumoniae. Meth. Enzymol. 358, 277-288 (2002)

3. Ciervo, A., Visca, P., Petrucca, A., Biasucci, L.M., Maseri, A., Cassone, A.: Antibodies to 60 -kilodalton heat shock protein and outer membrane protein 2 of Chlamydia pneumoniae in patients with coronary heart disease. Clin. Diagn. Lab. Immunol. 9, 66-74 (2002)

4. Kumar, S., Hammerschlag, M.R.: Acute respiratory infection due to Chlamydia pneumoniae: current status of diagnostic methods. Clin. Infect. Dis. 44, 568-576 (2007)

5. Boman, J., Hammerschlag, M.R.: Chlamydia pneumoniae and atherosclerosis: critical assessment of diagnostic methods and relevance to treatment studies. Clin. Microbiol. Rev. 15, 1-20 (2002)

6. Maass, M., Gieffers, J., Krause, E., Engel, P.M., Bartels, C., Solbach, W.: Poor correlation between microimmunofluorescence serology and polymerase chain reaction for detection of vascular Chlamydia pneumoniae infection in coronary artery disease patients. Med. Microbiol. Immunol. 187, 103-106 (1998)

7. Sanchez-Campillo, M., Bini, L., Comanducci, M., Raggiaschi, R., Marzocchi, B., Pallini, V., Ratti, G.: Identification of immunoreactive proteins of Chlamydia trachomatis by western blot analysis of a two- dimensional electrophoresis map with patient Sera. Electrophoresis 20, 2269-2279 (1999)

8. Bunk, S., Susnea, I., Rupp, J., Summersgill, J.T., Maass, M., Stegmann, W., Schrattenholz, A., Wendel, A., Przybylski, M., Hermann, C.: Immunoproteomic identification and serological responses to novel chlamydia pneumoniae antigens that are associated with persistent C. pneumoniae infections. J. Immunol. 180, 5490-5498 (2008)

9. Neuhoff, V., Arold, N., Taube, D., Ehrhardt, W.: Improved staining of proteins in polyacrylamide gels including isoelectric focusing gels with clear background at nanogram sensitivity using coomassie brilliant blue G-250 and R-250. Electrophoresis 9, 255-262 (1988)

10. Mortz, E., Vorm, O., Mann, M., Roepstorff, P.: Identification of proteins in polyacrylamide gels by mass spectrometric peptide mapping combined with database search. Biol. Mass Spectrom. 23, 249-261 (1994)

11. Wehrl, W., Brinkmann, V., Jungblut, P.R., Meyer, T.F., Szczepek, A.J.: From the inside out-processing of the chlamydial autotransporter PmpD and its role in bacterial adhesion and activation of human host cells. Mol. Microbiol. 51, 31-334 (2004)

12. Shaw, A.C., Vandahl, B.B., Larsen, M.R., Roepstorff, P., Gevaert, K., Vandekerckhove, J., Christiansen, G., Birkelund, S.: Characterization of a secreted chlamydia protease. Cell. Microbiol. 4, 411424 (2002) 